\title{
Construction of Semantic Navigation System of Maritime Accidents Based on Linked Data
}

\author{
Wei Guan ${ }^{1, a^{*}}$ and Yiduo Liang ${ }^{2, b}$ \\ ${ }^{1}$ Research and Training Center, Dalian Neusoft University of Information, China \\ ${ }^{2}$ Department of Information Management, Dalian Neusoft University of Information, China \\ aguanwei@neusoft.edu.cn, ${ }^{b}$ liangyiduo@neusoft.edu.cn \\ * The Corresponding Author
}

Keywords: Ontology; Maritime accident; Publishing of linked data; Semantic navigation

\begin{abstract}
In order to construct a semantic navigation system of maritime accidents based on linked data, the authors adopted the methods of ontology, which is the core technology of semantic Web, and linked data. An ontology model was firstly established of maritime accidents, which is used to describe cases. Then a publishing framework of linked data of maritime accidents was put forth, based on which the semantic navigation system was constructed. Meanwhile, mutual access is made possible through semantic links. The results of the paper demonstrate that linked data enjoys substantial advantages in semantic integration of heterogeneous data sources and sets the stage for the semantic interoperability of different sources.
\end{abstract}

\section{Introduction}

With the growth of China's shipping industry, there have arisen numbers of maritime accidents. It makes substantial sense to collect and sort out the information on the accidents and to enhance the security education of maritime affairs and intensify the regulation of maritime transportation. While some information systems have been established in this regard such as the Framework of the Collaborative System of Water Transportation Accidents Investigation and Handling put forth by Sang Lingzhi et al. from Research Centre of Smart Transportation System of Wuhan University of Technology[1] and the Local Platform of Maritime Information Based on the J2EE Technology by Xiao Zhi from University of Electronic Science and Technology of China[2]. However, the authors of the present paper found after analysis that the above systems only provide the matching and browsing of accident data through the input of key words; they fail to furnish further navigation semantically to other objects linked to the accidents in question. As a result, a more accurate, comprehensive and objective understanding of accidents cannot be achieved.

Tim Berners-Lee proposed the concept of linked data[3] in July of 2006, appealing to researchers from China and other countries as a lightweight implementation of semantic Web[4]. Currently in China, the research into this type of technology focuses on library science and informatics. A library acts as the publisher, verifier of reliability and consumer of linked data as well as the organizer and coordinator of linked data application[5]. In other fields, however, the technology is rarely used. One of the major reasons is that in the area of informatics, huge amounts of digital resources have been stored up over years of development so that rich high-quality data is accessible. Featuring unified data model and semantic link, linked data offers a brand-new insight to the researchers in other fields.

Based on the above analysis, the authors of the paper intended to introduce linked data to the investigation of maritime accidents for a combination. An ontology model was firstly established for maritime accidents, which is used to describe cases. Then a framework was put forth to publish linked data, based on which a semantic navigation system was constructed, demonstrating advantages in semantic integration of heterogeneous data sources. Finally, the conclusion was reached. 


\section{Ontology Model of Maritime Accidents}

The concept of ontology has a long history with different definitions. The authors of the present paper referred to the basic model put forth by AIFB Institute of Karlsruher Institute of Technology (KIT)[6] and extended it to construct an ontology model for maritime accidents research. The procedures of constructing the maritime accidents ontology are as follows:

Step (1):Constructing $C_{\text {accident }}$

The set of concepts $\left(C_{\text {accident }}\right)$ is used for the common concepts or classes in a specific area. A subject is a complete summarization of the terminology of a certain field, which is highly authoritative after being logically organized and refined by experts over years[7]. Ships are the subjects in maritime accidents. The class of ships was constructed according to the classification in Chinese Thesaurus of Communication[8]. The maritime accidents are grouped according to types and grades. The authors constructed the class of maritime accidents based on the descriptions in Measures on Statistics of Water Transportation Accidents[9]. Additionally, other necessary concepts were constructed based on analysis of the gathered information of maritime accidents. The $C_{\text {accident }}$ goes as follows:

$C_{\text {accident }}=$ MarineDomain, ShipDomain, MerchantShip, MilitaryVessel, ScienceShip, MarineType, CollisionAccident, EmeraldSea, MarineGrade, BigAccident, GeneralAccident, CrewDomain, CargoDomain, AccidentSiteDomain, ShipCompany, ...\}

Step (2): Constructing $R_{\text {accident }}$

$R_{\text {accident }}$ uses object property to describe the specific relations between concepts. The $R_{\text {accident }}$ for maritime accidents is as follows:

$R_{\text {accident }}=\{$ hasAccidentType, collision, isHappento, hasCrew, AccidentSite, ... $\}$

To describe the relationship between the concepts of maritime accidents and typed literal, the authors extended $R_{\text {accident }}$ by data property to be $R_{\text {accident-data }}$ :

$R_{\text {accident-data }}=\{$ AccidentTime, SeriousInjuries, AccidentDescription, DirectEconomicLosses, ... $\}$

Step (3): Constructing 5

$\sqsubseteq$ shows the hierarchy among the domain concepts. The partial order relationship of concepts for maritime accidents was constructed as follows:

$\sqsubseteq_{\text {accident }}=\{\langle$ ShipDomain, MarineDomain $\rangle,\langle$ MerchantShip, ShipDomain $\rangle,\langle$ MarineType, MarineDomain>, <CollisionAccident, MarineType>, <MarineGrade, MarineDomain>, $<$ BigAccident, MarineGrade>, <CrewDomain, MarineDomain>, <CargoDomain, MarineDomain>,

$<$ AccidentSiteDomain, MarineDomain $>,<$ ShipCompany, MarineDomain $>, \ldots\}$

Step (4): Constructing $\sigma$

The authors mapped the maritime accidents into the subset of $C_{\text {accident }} \times C_{\text {accident }}$ and the function came out as follows:

$\sigma_{\text {accident }}=\{\sigma$ (hasAccidentType $)=\{\langle$ ShipDomain, MarineType $\rangle\}, \sigma$ (collision $)=\{\langle$ ShipDomain, ShipDomain $>\}, \sigma$ (isHappento $)=\{\langle$ MarineType, ShipDomain $>\}, \sigma$ (hasCrew) $=\{\langle$ ShipDomain, CrewDomain $>\}, \sigma$ (AccidentSite $)=\{<$ MarineType, AccidentSiteDomain $>\}, \ldots\}$

Step(5): Constructing $A$

The set of axioms describes the truths and rules in a certain area to be used for reasoning. $A$ for maritime accidents is as follows:

$A=\{(? \mathrm{x}$ rdf:type maritime:MarineType), (?y rdf:type maritime:CargoShip), (?z rdf:type maritime:ShipDomain), (?y maritime:collision ?z), (?x maritime:SeriousInjuries ?a), ge(?a,1), notEqual(?y,?z)->(?x maritime:isType maritime:CollisionAccident)...\}

The authors of the paper formalize the ontology model in order to make it machine-friendly[10]. Protégé[11], an ontology editor developed by Stanford University, constructs simple and complex ontological applications through a structure based on plug-ins and is deemed as the most widely used visualized modelling tool for semantic Web. Protégé utilizes OWL as a language for description so that it enjoys a satisfactory logic basis and supports reasoning effectively. OWL statements can be automatically generated on the ontology model of maritime accidents. 


\section{Semantic Annotation of Shipping Examples}

The Maritime Accident Ontology Model provides metadata describing the instances of maritime accidents. In order to publish the related data of maritime accidents, it is necessary to obtain information on maritime accidents. The official website of the Maritime Safety Administration of the People's Republic of China and the Maritime Safety Administration of different provinces of China provide reports on maritime accidents. These official websites are the relatively authoritative source of maritime accident information and it can be accessed by visiting the above websites. Take a collision accident on the official website of Shandong Maritime Safety Administration as an example[12], according to the constructed maritime accident ontology model, the OWL description of the collision accident is generated. The original collision accident information on the website is illustrated in Figure 1.

Table 1 The Collision Information of the "Jintai 66" and "COSCO Fishing No.2"

\begin{tabular}{|c|l|}
\hline $\begin{array}{c}\text { Name of the } \\
\text { Accident }\end{array}$ & The Collision Between "Jintai 66" and "Zhongyuan Fishing 2" \\
\hline $\begin{array}{c}\text { Time of } \\
\text { Accident }\end{array}$ & $\begin{array}{l}2014-08-05 \\
\text { August 5, 2014 }\end{array}$ \\
\hline $\begin{array}{c}\text { Location of the } \\
\text { Accident }\end{array}$ & $\begin{array}{l}\text { 20 nautical miles east of Shidao port (under the jurisdiction of the } \\
\text { Weihai City, Shandong Province) }\end{array}$ \\
\hline $\begin{array}{c}\text { Geographic } \\
\text { Coordinates }\end{array}$ & $36^{\circ} 51.6^{\prime} \mathrm{N}, 122^{\circ} 51.2^{\prime} \mathrm{E}$ \\
\hline $\begin{array}{c}\text { Vessel 1 in the } \\
\text { Accident }\end{array}$ & $\begin{array}{l}\text { Jintai 66, under the jurisdiction of "Taizhou Jintai Shipping Co., Ltd., 16 } \\
\text { people on board }\end{array}$ \\
\hline $\begin{array}{c}\text { Vessel } 2 \text { in the } \\
\text { Accident }\end{array}$ & $\begin{array}{l}\text { COSCO Fishing No. 2, under the jurisdiction of CNFC Overseas } \\
\text { Fisheries Co., Ltd., 27 people on board }\end{array}$ \\
\hline $\begin{array}{c}\text { Description of } \\
\text { the Accident }\end{array}$ & $\begin{array}{l}\text { The second cabin on the port side of "Jingtai 66" was damaged and } \\
\text { flooded. The bow of "COSCO Fishing No. 2" was damaged and flooded } \\
\text { too. Through coordination, Vessel No.112 and No. 203 of Beihai } \\
\text { Rescue Bureau, Towboat No. 18 of Shidao Port, the passing merchant } \\
\text { ships "Huijinqiao No. 83", and "Xinmingfa No.127" came to rescue. } \\
\text { The two damaged vessels arrived Shidao Port safely respectively. Upon } \\
\text { arrival at Shidao Port, 43 people on both vessels were safely rescued. }\end{array}$ \\
\hline
\end{tabular}

Based on the constructed maritime accident ontology vocabulary, and reusing some other ontology words, the OWL statement fragments of the maritime accident examples are obtained as follows:

$<!$ - statement fragments of the maritime accident examples -->

<owl:NamedIndividual rdf:about="\&maritimeontology; Jintai 66 collided with COSCO Fishing No. 2 ">

<rdf:type rdf:resource=" \&maritimeontology;MarineType"/>

$<$ rdfs:label> Jintai 66 collided with COSCO Fishing No. 2</rdfs:label>

$<$ SeriousInjuries $>0</$ SeriousInjuries $>$

$<$ AccidentDescription>

"The second cabin on the port side of "Jingtai 66" was damaged and flooded, The bow of "COSCO Fishing No. 2" was damaged and flooded. ......

$</$ AccidentDescription>

$<$ wgs84_pos:long $>122^{\circ} 51.2^{\prime}</$ wgs84_pos:long $>$

$<$ wgs 84 _pos:lat $>36^{\circ} 51.6^{\prime}</$ wgs 84 _pos:lat $>$

<AccidentTime>2014-08-05</AccidentTime>

<isHappento rdf:resource="\&maritimeontology; COSCO Fishing No.2"/>

<AccidentSite rdf:resource="\&maritimeontology; 20 nautical miles east of Shidao port"/> 
<isHappento rdf:resource="\&maritimeontology; Jintai66"/> $</$ owl:NamedIndividual $>$

\section{Framework for the Publishing of Linked data on Maritime Accidents}

In order to achieve the access based on the semantic link between data, the above maritime accident instances are published as linked data in order to get a wider range of data browsing based on semantics and potential value-added link. The framework of the linked data publication proposed in this article is shown in Figure 1.

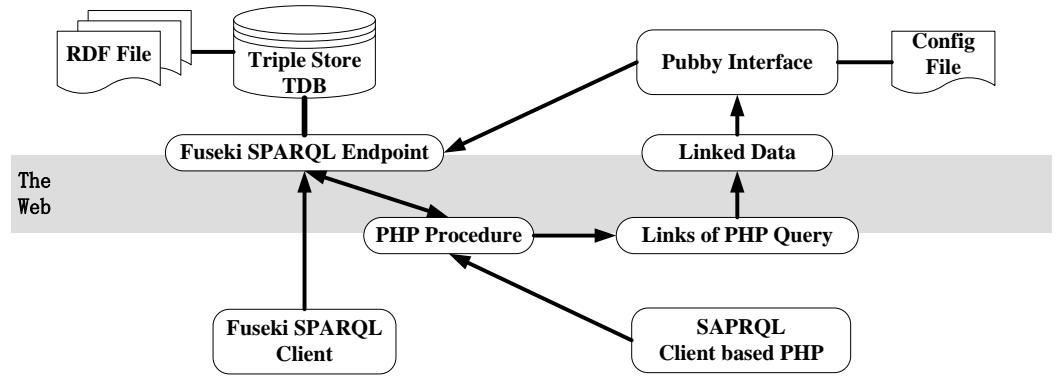

Figure 1. The publishing framework for the linked data of maritime accidents

In the above framework, TDB is used as a triad memory to store RDF data, and Fuseki is used as the access interface for SPARQL Endpoint to provide a RDF. At the same time, in order to realize the two mechanisms of redirection and content negotiation in the linked data, the Tomcat based Pubby application is placed at the front end of Fuseki, which provides the standard access interface of the linked data. In order to deal with linked data query and browse the process more flexibly, the author uses PHP scripting language to develop the SPARQL query template and uses ARC2 to interact with triad, Fuseki, SPARQL and Endpoint, while using PHP scripting language to process and display the query to ensure that the page accessibility of the linked data.

\section{Construction of Semantic Navigation System of Maritime Accidents}

The advantages of linked data by adding more links can realize the semantic relationship between different resources, which can be browsed to get more knowledge in various heterogeneous data resources, and this can't be realized by the traditional document with hyperlinks.

To illustrate the process of semantic navigation, a partial semantic navigation map based on associated links is shown below in Figure 2. 


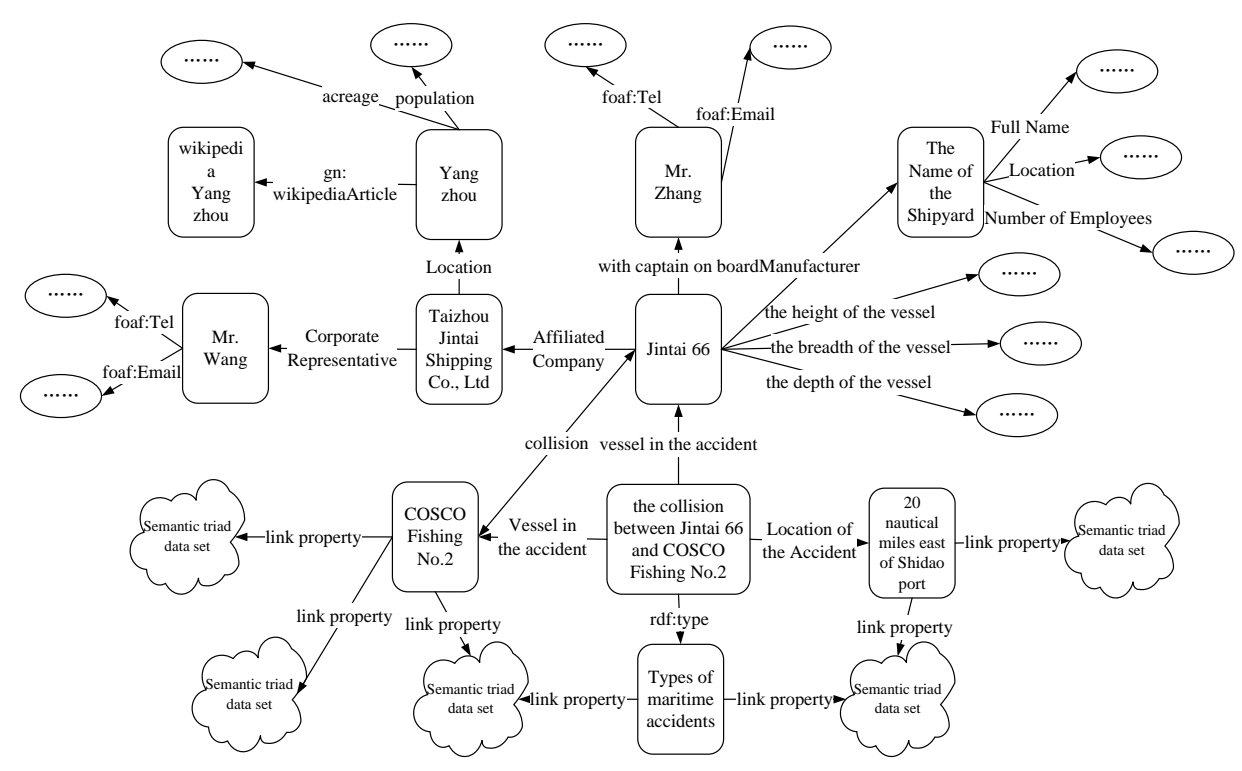

Figure 2. Semantic navigation based on associated links

From the figure above, Jintai 66, one of the vessels involved in the "Collision Between Jintai 66 and COSCO Fishing No.2" was Jintai 66. Click the URI of "Jintai 66" and then it can be switched to the linked data page of Jintai 66. By doing this, the semantic navigation is realized from the accident to vessel itself. In this page, some data to the properties of the vessel itself, such as height, breadth, depth of the vessel can be browsed. At the same time, by analogy, objects from the accident vessel can be navigated to vessel producers, captains, affiliated companies and other objects. The company can be further navigated to the company's legal representative and location of the company, so as to achieve the semantic navigation based on RDF diagram mode. In addition to the internal semantic navigation for these data sets, more external data set of semantic navigation can be achieved by adding more external links.

\section{Conclusion}

Based on the construction of maritime accident ontology and examples, this paper puts forward the releasing framework of linked data for maritime accidents, and realizes the construction of semantic navigation for maritime accidents, and shows the important value of linked data in realizing semantic integration of heterogeneous data sources.

\section{References}

[1] Z.L. Zhang: Chinese Navigation, Vol. 36 (2013) No.1, p.89. (In Chinese)

[2] Z. Xiao: Design and Implementation of Local Maritime Information Platform Based on Java EE (M.S., University of Electronic Science and Technology of China, China 2012), p.1.(In Chinese)

[3] Information on http://www.w3.org/DesignIssues/LinkedData.html

[4] C.J. Xia: Journal of Chinese Library Science, Vol. 38(2012) No.1, p.45.(In Chinese)

[5] D.Q. Chen: Library Construction, (2014) No. 3, p.17.(In Chinese)

[6] G. Stumme: The Karlsruhe View on Ontologies (Universitat Karlsruhe(TH), Karlsrube 2003).

[7] A. M. Tang: Modern Library Information Technology, Vol. 21(2005) No.4, p.1. (In Chinese)

[8] Ministry of Transport of the People's Republic of China: Chinese Thesaurus of Communication(Ministry of Communications Research Institute, China 2007). (In Chinese)

[9] Information on http://www.gov.cn/gongbao/content/2015/content_2799020.htm

[10] J. Zhai: Journal of The China Society for Scientific and Technical Information, Vol. 30(2011) No.11, p.1226. (In Chinese)

[11] Information on http://protege.stanford.edu/

[12] Information on http://sdmsa.gov.cn/sdmsa/news/201485937204532545325.shtml 